\title{
Practical considerations for implementing genomic information resources
}

\section{Experiences from eMERGE and CSER}

Luke V. Rasmussen'; Casey L. Overby 2,3; John Connolly4; Christopher G. Chute2; Joshua C. Denny5; Robert R. Freimuth ${ }^{6}$; Andrea L. Hartzler ${ }^{7}$; Ingrid A. Holm ${ }^{8,9}$; Shannon Manzi ${ }^{10}$; Jyotishman Pathak ${ }^{11}$; Peggy L. Peissig ${ }^{12}$; Maureen Smith ${ }^{13}$; Marc S. Williams ${ }^{3}$; Brian H. Shirts ${ }^{14}$; Elena M. Stoffel ${ }^{15}$; Peter Tarczy-Hornoch ${ }^{16}$; Carolyn R. Rohrer Vitek ${ }^{17}$; Wendy A. Wolf ${ }^{10,18,19}$; Justin Starren ${ }^{1}$

${ }^{1}$ Department of Preventive Medicine, Northwestern University Feinberg School of Medicine, Chicago IL;

${ }^{2}$ Department of Medicine, Johns Hopkins School of Medicine, Baltimore, MD;

${ }^{3}$ Genomic Medicine Institute, Geisinger Health System, Danville, PA;

${ }^{4}$ The Center for Applied Genomics, The Children's Hospital of Philadelphia, Philadelphia, PA;

${ }^{5}$ Vanderbilt University Medical Center, Vanderbilt University Medical Center, Nashville, TN;

${ }^{6}$ Department of Health Sciences Research, Division of Biomedical Statistics and Informatics, Mayo Clinic, Rochester, MN;

${ }^{7}$ Group Health Research Institute, Group Health Cooperative, Seattle, WA;

${ }^{8}$ Division of Genetics and Genomics and the Manton Center for Orphan Diseases Research, Boston Children's Hospital, Boston, MA;

${ }^{9}$ Department of Pediatrics, Harvard Medical School, Boston, MA;

${ }^{10}$ Department of Pharmacy and Division of Genetics \& Genomics, Boston Children's Hospital, Boston MA;

${ }^{11}$ Division of Health Informatics, Weill Cornell Medical College, Cornell University, New York City, NY;

${ }^{12}$ Biomedical Informatics Research Center, Marshfield Clinic Research Foundation, Marshfield Wl;

${ }^{13}$ Center for Genetic Medicine, Northwestern University Feinberg School of Medicine, Chicago IL;

${ }^{14}$ Department of Laboratory Medicine, University of Washington, Seattle, WA;

${ }^{15}$ Department of Internal Medicine, University of Michigan, Ann Arbor, MI;

${ }^{16}$ Departments of Biomedical Informatics and Medical Education; Pediatrics; Computer Science and Engineering, University of Washington Seattle WA;

${ }^{17}$ Center for Individualized Medicine, Mayo Clinic, Rochester, MN;

${ }^{18}$ Division of Genetics and Genomics, Boston Children's Hospital, Boston, MA:

${ }^{19}$ Unitio, Inc., Boston, MA;

\section{Keywords}

Electronic health records; genetics; clinical decision support; medicine; education

\section{Summary}

Objectives: To understand opinions and perceptions on the state of information resources specifically targeted to genomics, and approaches to delivery in clinical practice.

Methods: We conducted a survey of genomic content use and its clinical delivery from representatives across eight institutions in the electronic Medical Records and Genomics (eMERGE) network and two institutions in the Clinical Sequencing Exploratory Research (CSER) consortium in 2014. Results: Eleven responses representing distinct projects across ten sites showed heterogeneity in how content is being delivered, with provider-facing content primarily delivered via the electronic health record (EHR) $(n=10)$, and paper/pamphlets as the leading mode for patient-facing content $(n=9)$. There was general agreement $(91 \%)$ that new content is needed for patients and providers specific to genomics, and that while aspects of this content could be shared across institutions there remain site-specific needs ( $73 \%$ in agreement).

Conclusion: This work identifies a need for the improved access to and expansion of information resources to support genomic medicine, and opportunities for content developers and EHR vendors to partner with institutions to develop needed resources, and streamline their use - such as a central content site in multiple modalities while implementing approaches to allow for site-specific customization. 


\section{Correspondence to:}

Luke Rasmussen

Division of Health and Biomedical Informatics

Department of Preventive Medicine

Northwestern University Feinberg School of Medicine

750 North Lake Shore Drive

11th Floor, Rubloff Building

Chicago, IL 60611

Phone: 312-503-2823
Appl Clin Inform 2016; 7: 870-882

http://dx.doi.org/10.4338/ACI-2016-04-RA-0060

received: May 6, 2016

accepted: August 12, 2016

published: September 21, 2016

Citation: Rasmussen LV, Overby CL, Connolly J, Chute CG, Denny JC, Freimuth RR, Hartzler AL, Holm IA, Manzi S, Pathak J, Peissig PL, Smith M, Williams MS, Shirts BH, Stoffel EM, Tarczy-Hornoch P, Rohrer Vitek CR, Wolf WA, Starren J. Practical considerations for implementing genomic information resources - experiences from eMERGE and CSER. Appl Clin Inform 2016; 7: 870-882

http://dx.doi.org/10.4338/ACI-2016-04-RA-0060

Funding

The eMERGE Network was initiated and funded by NHGRI through the following grants: U01HG006828 (Cincinnati Children's Hospital Medical Center/Boston Children's Hospital); U01 HG006830 (Children's Hospital of Philadelphia); U01HG006389 (Essentia Institute of Rural Health, Marshfield Clinic Research Foundation and Pennsylvania State University); U01HG006382

(Geisinger Clinic); U01HG006375 (Group Health Cooperative/University of Washington); U01 HG006379 (Mayo Clinic); U01HG006380 (Icahn School of Medicine at Mount Sinai); U01HG006388 (Northwestern University); U01HG006378 (Vanderbilt University Medical Center); and U01HG006385 (Vanderbilt University Medical Center serving as the Coordinating Center).

The Clinical Sequencing Exploratory Research Program (CSER) was initiated and funded by NHGRI and the $\mathrm{NCl}$ through the following grants: U01 HG006485 (Baylor College of Medicine); U01 HG006500 (Brigham \& Women's Hospital); U01 HG006546 (Children's Hospital of Philadelphia); R01 HG006600 (Columbia University); U01 HG006492 (Dana-Farber Cancer Institute); UM1 HG007301 (HudsonAlpha Institute); UM1 HG007292 (Kaiser Permanente); UM1 HG006508 (University of Michigan); U01 HG006487 (University of North Carolina); U01 HG006507 (University of Washington); and U01 HG007307 (University of Washington serving as the Coordinating Center). 


\section{Background and Significance}

Although genetic information has been used in healthcare for many years, recent genomic advances have increased attention towards how genomic data may be more broadly used in healthcare, and what systems must be implemented to support this [1]. In particular, substantial growth in knowledge about the human genome has enabled subsequent application to pharmacogenomics (PGx) and targeted therapies, risk assessment, and diagnostic algorithms. As a result, there is an increased demand on physicians, nurses, pharmacists and other healthcare providers to integrate genetic results into the care plan, at a time when our understanding of the human genome is continually expanding.

While advances have been made in increasing the awareness of healthcare providers in this evolving field, providers still cite significant gaps in knowledge and level of comfort with respect to test ordering and applying results from genetics and genomics analyses [2-4], including PGx [5-9]. These gaps suggest the need for increased educational efforts in genomic medicine $[3,10]$, including formal education and training, as well as point-of-care decision support aids $[11,12]$. Education needs to be provided in a way that supports confident clinical decision making for all involved disciplines [12]. The impact extends to patients as well, with studies citing patients' lack of understanding of genetic and genomic results [13], but a desire to better understand management and prevention of genetic conditions [14]. An important consideration, therefore, is that information resources are made available to both providers and patients to support understanding and decision-making in the context of genomic and genetic results.

A variety of information resources on genetic testing, diagnoses and treatment options exist. These vary from institutional subscription services to others that are freely available. Likewise, these resources may provide a more comprehensive overview of a specific topic, or may be more targeted towards certain questions. Examples include patient-focused sites like Genetics Home Reference that provides an overview of genetics and gene-disease relationships [15], as well as provider-focused resources like GeneReviews $^{\oplus}$ [16] and the Clinical Pharmacogenetics Implementation Consortium (CPIC) guidelines [17] that provide actionable information to healthcare providers.

Several institutions have described their implementation of genomic medicine (GM, assessing risk for heritable conditions, such as hemochromatosis) and PGx (looking at one or more drug-gene combinations) projects [18-22]. Assessments of implementation strategies within the electronic Medical Records and Genomics (eMERGE) [23, 24] and Clinical Sequencing Exploratory Research (CSER) [25] networks have shown significant heterogeneity in how results are returned to physicians, pharmacists and patients. In spite of this variation, almost all implementations have included some form of information resources to help with the interpretation of genomic results [26-28]. For example, at some institutions results may be returned as text reports with embedded hyperlinks, or content from information resources copied directly into the report. Other institutions return structured laboratory results and link to specific external references via a standardized terminology code (e.g. LOINC) associated with the result. However, no work to date has focused on this use of information resources to support genomic medicine programs.

Surveying the experiences of institutions in the eMERGE and CSER networks, we explore how these sites use information resources within their genomic medicine programs. In addition, we evaluate how their strategies may support the improved access and expansion of information resources more broadly.

\section{Methods}

A survey was developed by three of the authors (LVR, CLO, JC) (available in Appendix A) to capture site-specific information about: a) current or future plans for delivering information resources, b) modalities for delivering information resources, c) current or planned use of internally and externally hosted resources, d) opinions on the quality and availability of information resources, and e) opinions on the generalizability of content contained in information resources. Questions differentiated between resources targeted to patients and healthcare providers to assess if similarities or differ- 
ences existed between these audiences. Validation or reliability testing was deemed unnecessary given the descriptive and qualitative nature of the survey.

Institutions in the eMERGE and CSER networks planned to implement PGx and/or GM projects. Institutions that anticipated different responses for these projects were asked to respond separately for PGx and GM. Given that institutions were in different states of planning and implementation for both PGx and GM projects, they were also instructed to only respond for the project(s) that they felt had an established plan for the delivery of information resources.

Survey questions related to the delivery of content asked respondents to distinguish between current and/or future plans to offer locally hosted content (that is, content delivered by a server or other resource directly under the institution's control), and remotely hosted content (that which is maintained by an external organization, such as a public website or commercial content provider). Questions related to modality for content management for patients and providers included four possible response options with an "Other" category. Choices included delivery: as a printed handout or pamphlet, from the EHR (which included information displayed in alerts to physicians and/or pharmacists, or template content included as part of the patient's after visit summary generated by the EHR), from a website or content management system delivering content as HTML pages (optionally including other multimedia), or as linked PDFs. For this study, the distinction between websites and PDFs, since PDFs are many times served via websites, is that the PDFs were static representations of content, whereas a website (or content management system) is a platform that offers the opportunity to provide dynamic or multimedia content. Likewise, the distinction between PDFs and paper printouts/pamphlets is that the PDF was delivered electronically, while the printout/pamphlet was made physically available. An additional delivery option of personal health record (PHR) or patient portal was added for patient-targeted content. The distinction between PHR/portal and CMS/website is that the former was configured or delivered through a PHR/portal (often dynamically), while the latter was typically a static content reference.

An e-mail request to complete the survey was sent to ten eMERGE and eight CSER institutions in September 2014, with the survey open for responses for one month. The e-mail was sent to one or two primary representatives at each eMERGE site that were active within the eMERGE EHR Integration (EHRI) workgroup. The decision to e-mail a select group instead of the entire EHRI workgroup mailing list was done to focus the request to those known to be primary stakeholders within their implementation project. Given a lack of similar knowledge about participating CSER sites, the decision was made to submit a similar request to the entire CSER EHR workgroup mailing list. One e-mail reminder was sent to the respective original distribution groups approximately two weeks before the survey closed. Survey responses were captured using Google Forms (Google, Mountain View, CA), and summarized with descriptive statistics. Aggregated results were discussed within the eMERGE EHR Integration workgroup. Discussion allowed for the expansion of ideas - such as details on how PGx and GM projects were being implemented at each site - and an opportunity to explore possible explanations for our findings related to the survey results. In addition, clarifying questions were sent to individual sites as needed. If an eMERGE institution provided a separate response for PGx and GM, the results were reviewed for similarity. If responses were the same across both projects, they were collapsed into a single response for that institution.

\section{Results}

In total, eight of ten eMERGE and two of eight CSER sites responded (55.6\% response rate). Where institutions provided two sets of responses for the same project, the institution was asked to review and provide a single, consensus response. Two eMERGE institutions provided separate responses each for PGx and GM projects. In review, one institution's pair of responses was identical across each of the projects and was consolidated back into a single response. This resulted in eleven responses across the ten institutions for analysis.

All eleven projects indicated that they were currently or had plans to deliver information resources authored by their institution as part of their project. Of these eleven projects that were delivering content to providers, eight indicated that they were currently providing content, and two indicated that they were planning to. One project representative did not provide a response. Respon- 
dents indicated that for six projects they were currently providing content to patients, and for five projects indicating they planned to do so. No response was provided for one project, and for another project there were conflicting answers regarding both providing and planning to provide content. Upon clarifying, we found that there was content offered, but that the project was planning to further enhance and expand the content.

Regarding modalities for how content was currently or planned to be delivered to providers, - Table 1 shows the overall responses. Project representatives had the opportunity to respond about multiple formats used, and so multiple responses were allowed. Across the projects, the majority used more than one mode of delivery to providers (median $=2, \mathrm{IQR}=1, \min =1, \max =4$ ). Delivering content in the EHR was the most common response $(n=10)$, followed by delivery via a website or content management system (CMS) $(n=6)$. Respondents indicated that for three projects "Other" modes of delivery were used, with two projects responding that they utilized e-mail, and another project "developed a course that can be accessed through our compliance education system", and was also exploring potential new approaches.

For content returned to patients (shown in $>$ Table 2), the majority of projects were providing or planned to provide content as printed handouts or pamphlets $(n=9)$, with content defined in the EHR or delivered through a PHR or patient portal as the next most frequent responses $(n=5)$. Of the five institutions using a PHR/portal to deliver content, two of those projects used both a PHR/portal and CMS/website. Respondents also indicated that for two projects "Other" modes of delivery were being used, indicating that they were actively exploring new and enhanced approaches such as "patient facing genomic test reports". The majority of projects also used more than one mode of delivery to patients (median=2, IQR=2, $\min =1, \max =4$ ).

- Figure 1a shows the intersection of projects that currently or plan to offer locally hosted content to providers compared to those currently or planning to offer remotely hosted content. The majority of projects had no plans to deliver remotely hosted content to providers, with three responding that they did currently and one planning to. Those sites currently utilizing remotely hosted content were all also providing locally hosted content. All eleven projects were providing or planned to provide locally hosted content. Figure $1 \mathrm{~b}$ shows the same graph for patient content, with similar trends. Only one project was providing remotely hosted content (and also providing remotely hosted physician content), one project was planning to provide remotely hosted content, and ten projects were or planned to provide locally hosted content.

- Figure 2 shows respondent's opinions on the need for new information resources, and transportability of content for providers and patients at other sites. Free-text comments are available in Appendix B. The largest area of agreement (providing a response in some degree of agreement) was regarding the need to develop new content ( $\$$ Figure $2 \mathrm{a}$ ), and that content is generalizable to other sites ( Figure $2 \mathrm{~d}$ ). The level of agreement was $91 \%$ across both questions, and was the same for both provider- and patient- facing information resources. The largest area of disagreement was regarding opinions about content specific to an institution ( $>$ Figure $2 \mathrm{c}$ ) with three projects (27\%) disagreeing that content for providers and patients is specific to an institution.

\section{Discussion}

These results illustrate the need to manage and deliver information resources for genomic medicine in a way that is effective for each institution, and to develop IT infrastructure that facilitates implementation into clinical care. Since genomic information is being more rapidly updated, there are additional challenges to ensure content is not stagnant. The differences in functionalities among EHRs also constitutes important barriers to the implementation of information resources and genomic medicine overall. Initiatives such as the HL7 Clinical Genomics group, [29] and the Global Alliance for Genomic Health (GA4GH) [30] have begun addressing these barriers through proposed standard representations of genomic results, which would allow EHRs and external systems to have a standard, computable definition for the exchange of genomic information. The ClinGen project has leveraged the OpenInfobutton approach initially developed as part of eMERGE [31] to allow searching across a large set of genomic resources [32]. Since OpenInfobutton is an HL7-compliant system and meets the EHR Meaningful Use standards [33] it is anticipated that this approach will allow 
local EHRs to access genomic resources in a context-sensitive manner. Furthermore, projects such as SMART on FHIR Genomics [34] have demonstrated the extension of native EHR functionality with optimized displays for genomic results, and the Displaying and Integrating Genetic Information Through the EHR (DIGITizE) Action Collaborative [35] has engaged multiple stakeholders, including EHR vendors, to empower clinical IT to support and implement genomics in the EHR. Building upon these existing efforts, additional work is still needed to focus on the integration of information resources into the EHR. This will allow central sources of knowledge to be managed to the benefit of everyone, rather than having each individual institution reinvent the wheel.

It is challenging to find effective ways of delivering genomic information to providers [36]. In this study, we see the majority of projects leveraging the EHR as a mode of delivery to providers. This most often involves configuring the EHR with a static version of the content, such as a brief summary statement in an alert window with links to supplemental information. Typically, the supplemental information resource also contains the summary statement, requiring separate updates (possibly across different groups), resulting in duplicated effort. Since genomic knowledge has the potential to evolve more rapidly, there is an added risk that genomic content in the EHR will become out of sync with that available in a supplemental resources external to the EHR. While this is currently managed manually, there is an opportunity for EHR vendors to create mechanisms such that EHR content (including brief summaries) could be dynamically composed from an external primary content repository while still allowing for local customization. For example, there may be an article written in a content system that describes what actions to take for a predicted clopidogrel poor metabolizer when writing a new prescription. A CDS alert in the EHR, instead of having a static recommendation entered, could send a request to the content system to retrieve and display the recommendation text. Processing context-specific requests is currently supported by the HL7 Infobutton Standard [37], and is supported by several EHRs to open a link to external resources. Currently, EHRs do not widely support this approach to retrieve and display dynamic content, although projects such as SMART CDS Hooks [38] have demonstrated approaches to provide customized, dynamic CDS results. Solutions in this area also requires content systems, such as a CMS or website hosted at an institution, to support the retrieval of dynamic content. In $>$ Figure 3, we illustrate how such a solution might be realized. By using a central genomic information repository to store content, annotated by context (e.g., if the article is for the patient or a provider, which genotype the content is for) requests may be made from multiple systems to receive targeted pieces of the relevant articles. For example, the prescribing recommendation for a predicted poor clopidogrel metabolizer could be shown in a CDS alert, and the same content could be visible within the context of a full article when browsing the content from an educational website. The continued exploration of this idea is an active area of research within eMERGE and ClinGen [39], which as noted has implemented resource searching using OpenInfobutton [32].

For delivering content to patients, fewer institutions utilized the EHR than when delivering to providers. Instead, sites generally provided paper handouts/pamphlets and/or an external CMS/ website for patients. This suggests the continued relevance of printed material, which may be explained if an institution was not able to fully leverage a patient portal to deliver content electronically, could not integrate an electronic option into the provider workflow, or wanted to engage patients in different formats that may be more accepted or preferred by the patient. Furthermore, adoption and use of patient portals varies across populations and is not ubiquitous [40-42], necessitating the use of other modalities. New methods of content delivery being explored within patient populations (indicated in the free text responses) may improve patient comprehension and retention.

We found few institutions providing remotely hosted content option for providers or patients. This may be caused by a variety of factors, such as an institutional preference to have control over hosted content and updates, or the choice to locally author content specific for the research programs that were the focus of the survey. As locally hosted content did not necessarily indicate locally generated content (the distinction being that a content provider providing a local installation of their content would be considered locally hosted), this does not preclude the need for externally developed content. Rather, it shows the importance of content providers delivering solutions that allow for local hosting or integration of their resources. 
Regarding the content that is available for genomic programs, the majority of respondents agreed about the need for development of new content to support both providers and patients. Indeed, subsequent to the survey one institution that had indicated it was exploring other options has developed complementary patient and provider facing interactive genomic test reports that can be accessed from the EHR and tethered patient portal through a locally developed application [43, 44]. Most respondents expressed some level of agreement that existing resources for both providers and patients were (or would be) used, although there was more agreement with provider-targeted resources specifically. Greater agreement with patient-targeted materials may be attributed to topics (such as specific drug-gene pairs) that are not covered by existing resources, or perception that existing resources are not appropriately targeted to patients or certain patient populations (e.g. pediatrics). This finding should encourage content developers to continue creation of new and refinement of existing resources - especially with the growing base of knowledge around genomic results.

An additional consideration is the opinion of respondents that the content for providers and patients is site-specific - that is, there exists some information within the content that requires local customization. This is consistent with our earlier assessment of content templates, in which we identified areas to enter institutional instructions or recommendations [31]. Given the research nature of the projects covered by the survey, local content might include local contact information regarding the research protocol or study staff, but comments within the survey noted the importance of customization for local workflow and culture, and the ability to control formatting or branding of the display. However, respondents also indicated a higher level of agreement that content for patients and providers is generalizable to other sites. While this finding initially seemed to contradict the opinion that content is site-specific, the reasoning is that much of the content is reusable in whole or with minor modifications/additions. That is, an entire information resource may not be reused in its entirety, given site-specific needs, but that many sections may be reused and others suppressed, synthesized or otherwise generalized for the institution. This highlights what is likely to become an ongoing tension as genomic medicine evolves. Specifically, it is not possible for most institutions to maintain and update a comprehensive genomic medicine information resource-favoring centralized resources. At the same time, both providers and patients want actionable information. Such actions tend to be site specific-favoring local resources. We believe this is an important finding, as it significantly alters the mindset of developing resources to compose sections as independent components of a document, and consider construction of documents as a combination of existing and new sections. Although external resources are and will continue to be used, their structure may be altered or optimized for reuse. Existing work in eMERGE has created a template that may contribute to sharing and reuse [31], however formal study is needed and is planned as future work.

Our study has several limitations. First, few CSER sites were represented in our sample (two of eight). We believe this is due to the project originating within the eMERGE network, and then later extending to CSER sites. Although there were a smaller number of CSER sites, we believe including CSER sites offers additional perspectives that may not otherwise have been seen. At the time of this survey, the eMERGE consortium was specifically focusing on incorporation of coded oligo-variant data and CDS, while the CSER consortium was focused on broader workflow and presentation issues related to clinical sequence data. Second, while results are reported by institution, the results represent the limited knowledge of specific individuals involved in genomic EHR research: the results are not official responses for the organization as a whole and were not the result of a comprehensive survey of every individual and group involved with the EHR at the organization. However, as previously noted the respondents were those involved within their respective genomic implementation project and qualified to respond within that context. Third, the results do not include outcomes of each respondent's approach. Some sites are collecting end user assessments, and evaluation and reporting of these findings are considered as future work. Finally, the respondents represented institutions that were specifically funded to implement and evaluate genomic results returned at the point of care. While not all organizations are currently capable of returning genomic results, we believe these findings can drive future innovation to make information resources more accessible to any practice, and provide a practical understanding of how information resources have been delivered to date. 


\section{Conclusions}

Based on the experience of several institutions engaged in the return of genomic results to patients and providers, there is an identified need for the continued development and expansion of information resources in support of genomic medicine. In addition, opportunities exist for content and EHR vendors to better integrate platforms to reduce the burden of maintaining the same or similar content in multiple locations. Continued focus in this area can provide downstream benefits as the landscape of genomic medicine advances.

\section{Clinical Relevance Statement}

Multiple information resources to support genomic medicine are available, however institutions may have additional site-specific needs. Although multiple modalities of resources may be used, current systems do not always support reducing duplication of effort in their maintenance. Organizations should plan accordingly to support these resources until improvements are made in the supporting infrastructure.

\section{Conflicts of Interest}

The authors declare that they have no conflicts of interest in the research.

\section{Protection of Human and Animal Subjects}

Human subjects were not included in this project. 


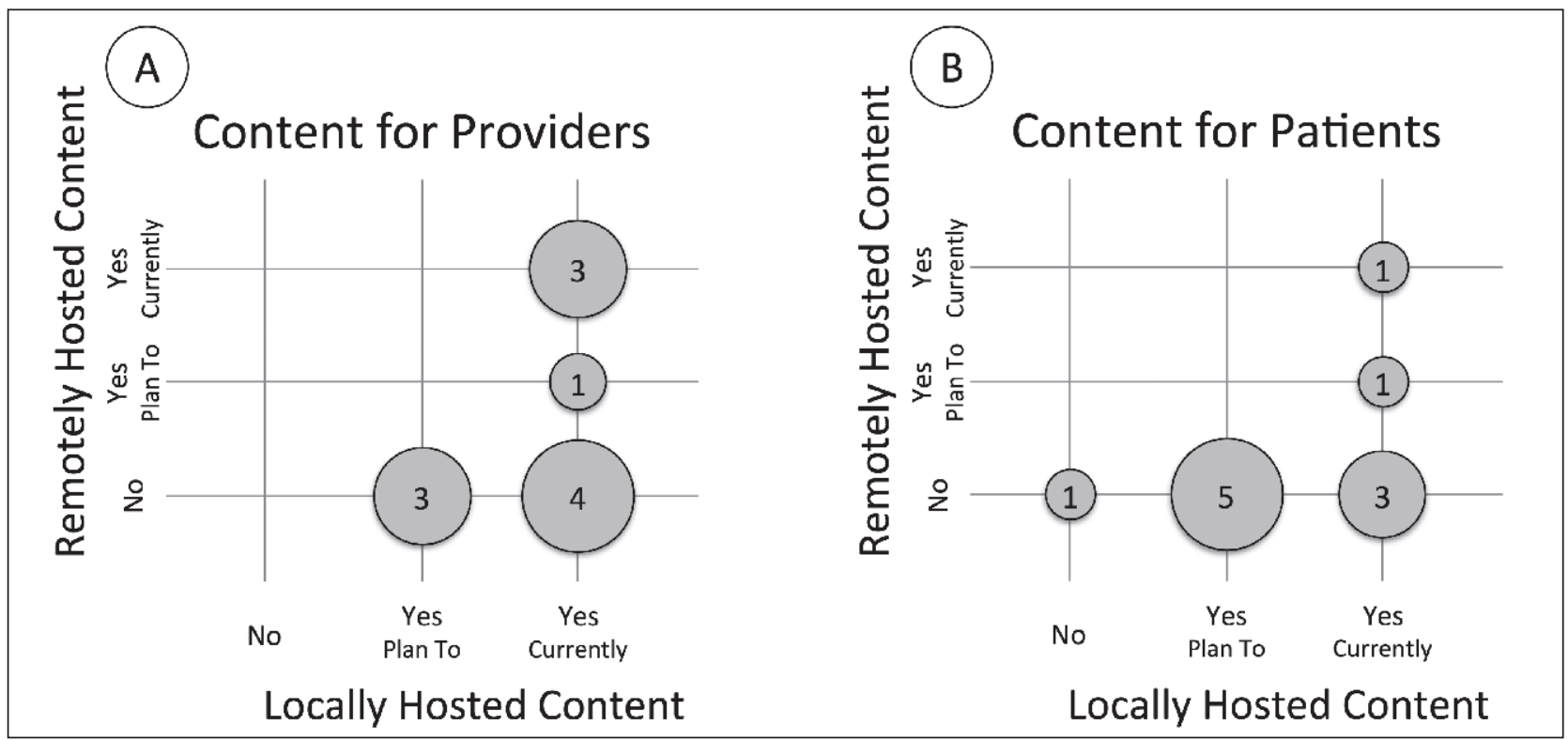

Fig. 1 Project responses regarding the use of remotely and/or locally hosted content for (A) providers, and (B) patients.

(a) New content needs to be developed to support decisions for: Providers Patients

(b) Existing resources provide the content needed to support decisions, and is/will be used at our site for: Providers Patients

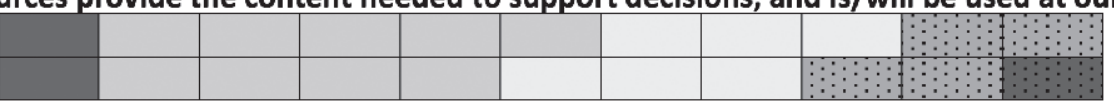

(c) Content to support decisions is site-specific for: Providers Patients

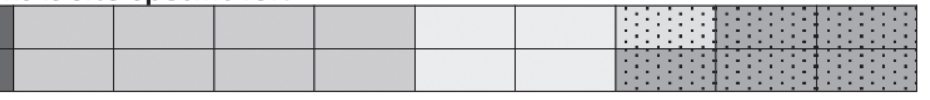

(d) Content to support decisions is generalizable to other sites for:

Providers

Patients

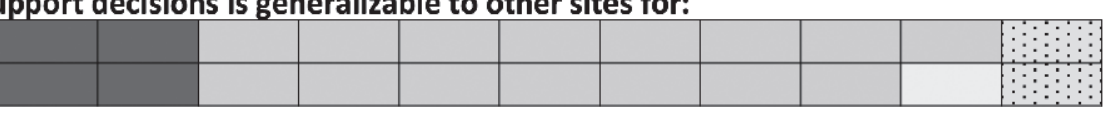

\begin{tabular}{|c|c|c|c|c|c|}
\hline & & & & & \\
\hline Strongly & Moderately & Slightly & Slightly & Moderately & Strongly \\
\hline \multicolumn{3}{|c|}{ Agree } & \multicolumn{3}{|c|}{ Disagree } \\
\hline
\end{tabular}

Fig. 2 Level of agreement regarding the state of existing content and resources, and how specific or generalizable information is across institutions (each block represents one response, $n=11$ ). 


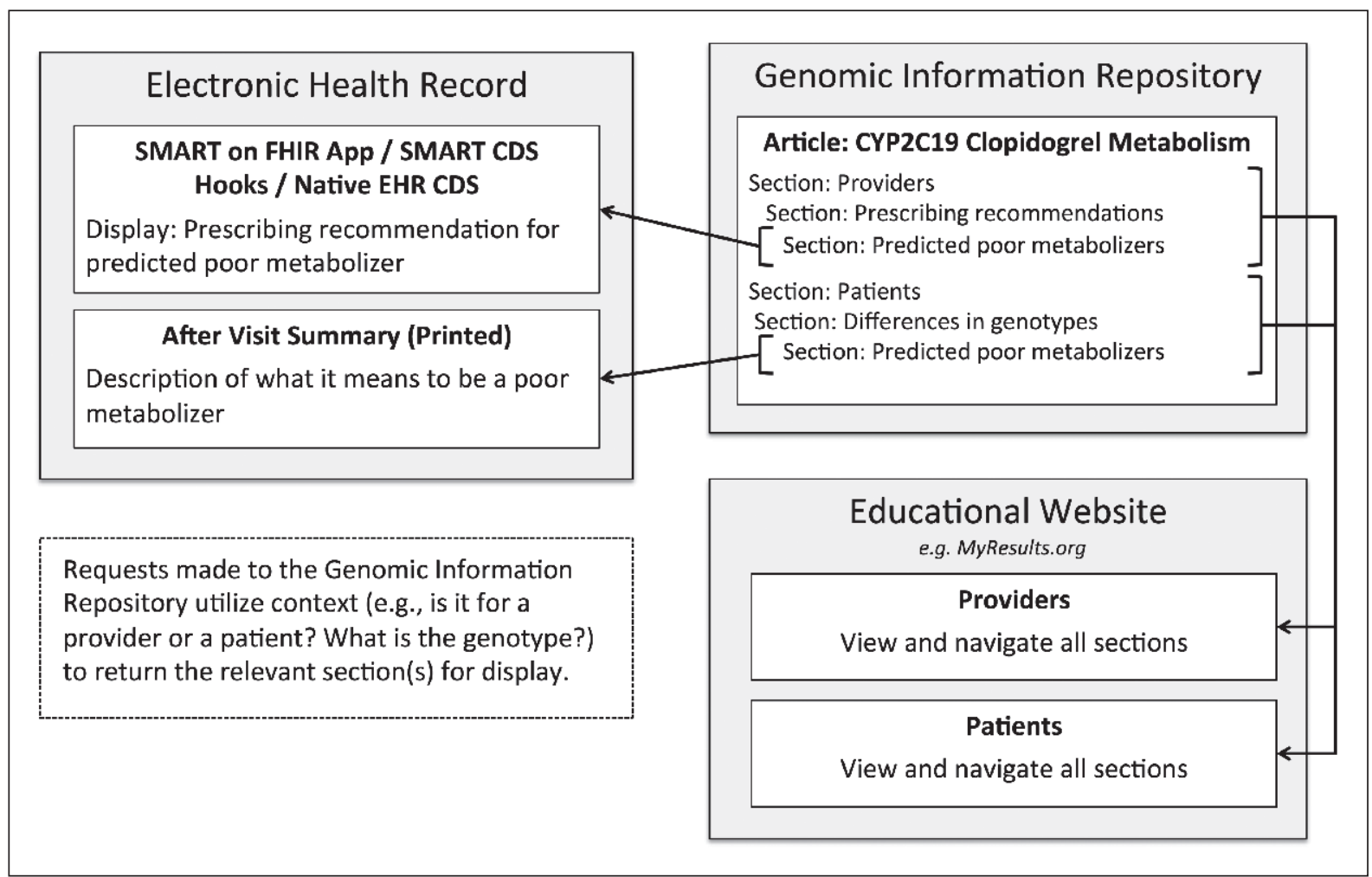

Fig. 3 Proposed environment where multiple systems (clinical decision support, educational websites, etc.) may use a contextual request to get information from a centralized genomic information repository. 
Table 1 Institutional responses to how they are currently, or plan to be, returning content to providers. Responses are related to pharmacogenomics (PGX) and/or Genomic Medicine (GM) scenarios as noted next to the institution. $\mathrm{EHR}=$ Electronic health record; $\mathrm{CMS}=$ content management system.

\begin{tabular}{|c|c|c|c|c|c|c|}
\hline & $\begin{array}{l}\text { Paper/ } \\
\text { Pamphlets }\end{array}$ & $\begin{array}{l}\text { Defined } \\
\text { in EHR }\end{array}$ & $\begin{array}{l}\text { External } \\
\text { PDFs }\end{array}$ & $\begin{array}{l}\text { CMS/ } \\
\text { Website }\end{array}$ & Other & Total \\
\hline Boston Children's Hospital (PGx/GM) & Y & Y & & Y & & 3 \\
\hline $\begin{array}{l}\text { Children's Hospital of Philadelphia } \\
\text { (PGX/GM) }\end{array}$ & & Y & & Y & & 2 \\
\hline Geisinger Health System (PGx) & & Y & & & Y & 2 \\
\hline Geisinger Health System (GM) & & Y & Y & Y & Y & 4 \\
\hline Group Health (PGx) & Y & & & & & 1 \\
\hline Marshfield Clinic (PGx/GM) & & Y & Y & & & 2 \\
\hline Mayo Clinic (PGx/GM) & Y & Y & & Y & Y & 4 \\
\hline Northwestern University (PGx/GM) & & Y & & Y & & 2 \\
\hline Vanderbilt University (PGx/GM) & Y & Y & & Y & & 3 \\
\hline University of Michigan (PGx/GM) & & Y & & & & 1 \\
\hline $\begin{array}{l}\text { University of Washington (CSER) (PGX/ } \\
\text { GM) }\end{array}$ & Y & Y & & & & 2 \\
\hline Totals & 5 & 10 & 2 & 6 & 3 & \\
\hline
\end{tabular}

Table 2 . Institutional responses to how they are currently, or plan to be, returning content to patients. Responses are related to pharmacogenomics (PGx) and/or Genomic Medicine (GM) scenarios as noted next to the institution. $E H R=$ Electronic health record; PHR = personal health record; CMS = content management system.

\begin{tabular}{|c|c|c|c|c|c|c|c|}
\hline & $\begin{array}{l}\text { Paper/ } \\
\text { Pamphlets }\end{array}$ & $\begin{array}{l}\text { Defined } \\
\text { in EHR }\end{array}$ & $\begin{array}{l}\text { External } \\
\text { PDFs }\end{array}$ & $\begin{array}{l}\text { PHR/ } \\
\text { Portal }\end{array}$ & $\begin{array}{l}\text { CMS/ } \\
\text { Website }\end{array}$ & Other & Total \\
\hline $\begin{array}{l}\text { Boston Children's Hospital (PGXI } \\
\text { GM) }\end{array}$ & Y & & & & & & 1 \\
\hline $\begin{array}{l}\text { Children's Hospital of Philadelphia } \\
\text { (PGx/GM) }\end{array}$ & Y & & & & Y & & 2 \\
\hline Geisinger Health System (PGx) & Y & & Y & & & Y & 3 \\
\hline Geisinger Health System (GM) & Y & & Y & & & Y & 3 \\
\hline Group Health (PGx) & & Y & & Y & & & 2 \\
\hline Marshfield Clinic (PGx/GM) & Y & & Y & & & & 2 \\
\hline Mayo Clinic (PGx/GM) & Y & Y & & Y & Y & & 4 \\
\hline Northwestern University (PGx/GM) & Y & Y & & Y & Y & & 4 \\
\hline Vanderbilt University (PGx/GM) & Y & Y & & Y & & & 3 \\
\hline University of Michigan (PGx/GM) & & Y & & & & & 1 \\
\hline $\begin{array}{l}\text { University of Washington (CSER) } \\
\text { (PGX/GM) }\end{array}$ & Y & & & Y & & & 2 \\
\hline Totals & 9 & 5 & 3 & 5 & 3 & 2 & \\
\hline
\end{tabular}




\section{References}

1. Green ED, Guyer MS. Charting a course for genomic medicine from base pairs to bedside. Nature 2011 ;470(7333): 204-213.

2. Klitzman R, Chung W, Marder K, Shanmugham A, Chin L, Stark M, et al. Attitudes and Practices Among Internists Concerning Genetic Testing. Journal of Genetic Counseling 2013; 22(1): 90-100.

3. Calzone KA, Cashion A, Feetham S, Jenkins J, Prows CA, Williams JK, et al. Nurses transforming health care using genetics and genomics. Nurs Outlook. 2010; 58(1): 26-35.

4. Selkirk CG, Weissman SM, Anderson A, Hulick PJ. Physicians ' preparedness for integration of genomic and pharmacogenetic testing into practice within a major healthcare system. Genet Test Mol Biomarkers 2013; 17(3): 219-225.

5. Dodson C. Knowledge and attitudes concerning pharmacogenomics among healthcare professionals. Personalized Medicine 2011; 8(4): 421-428.

6. Johansen Taber KA, Dickinson BD. Pharmacogenomic knowledge gaps and educational resource needs among physicians in selected specialties. Pharmgenomics Pers Med 2014; 7: 145-162.

7. Overby CL, Erwin AL, Abul-Husn NS, Ellis SB, Scott SA, Obeng AO, et al. Physician Attitudes toward Adopting Genome-Guided Prescribing through Clinical Decision Support. J Pers Med 2014; 4(1): 35-49.

8. Stanek EJ, Sanders CL, Taber KAJ, Khalid M, Patel A, Verbrugge RR, et al. Adoption of Pharmacogenomic Testing by US Physicians: Results of a Nationwide Survey. Clin Pharmacol Ther 2012; 91(3): 450-458.

9. Katsanis SH, Minear MA, Vorderstrasse A, Yang N, Reeves JW, Rakhra-Burris T, et al. Perspectives on genetic and genomic technologies in an academic medical center: the duke experience. J Pers Med 2015; 5(2): $67-82$.

10. Passamani E. Educational challenges in implementing genomic medicine. Clin Pharmacol Ther 2013; 94(2): 192-195.

11.Devine EB, Lee CJ, Overby CL, Abernethy N, McCune J, Smith JW, et al. Usability evaluation of pharmacogenomics clinical decision support aids and clinical knowledge resources in a computerized provider order entry system: a mixed methods approach. Int J Med Inform 2014; 83(7): 473-483.

12. Overby CL, Devine EB, Abernethy N, McCune JS, Tarczy-Hornoch P. Making pharmacogenomic-based prescribing alerts more effective: A scenario-based pilot study with physicians. J Biomed Inform 2015; 55: 249-259.

13. Reiff M, Bernhardt BA, Mulchandani S, Soucier D, Cornell D, Pyeritz RE, et al. „What does it mean?“: uncertainties in understanding results of chromosomal microarray testing. Genet Med 2012; 14(2): 250-258.

14. Houfek JF, Soltis-Vaughan BS, Atwood JR, Reiser GM, Schaefer GB. Adults' perceptions of genetic counseling and genetic testing. Appl Nurs Res 2015; 28(1): 25-30.

15. National Library of Medicine. Genetics Home Reference. [July 30, 2016]; Available from: http://ghr.nlm.nih.gov/.

16. Pagon R, Adam M, Ardinger H, Wallace S, Amemiya A, Bean L, et al. GeneReviews ${ }^{\bullet}$. Seattle, WA: University of Washington, Seattle; [July 30, 2016]; Available from: http://www.ncbi.nlm.nih.gov/books/ NBK1116/.

17. Caudle KE, Klein TE, Hoffman JM, Muller DJ, Whirl-Carrillo M, Gong L, et al. Incorporation of pharmacogenomics into routine clinical practice: the Clinical Pharmacogenetics Implementation Consortium (CPIC) guideline development process. Curr Drug Metab 2014; 15(2): 209-217.

18. Gottesman O, Scott SA, Ellis SB, Overby CL, Ludtke A, Hulot JS, et al. The CLIPMERGE PGx Program: clinical implementation of personalized medicine through electronic health records and genomics-pharmacogenomics. Clin Pharmacol Ther 2013; 94(2): 214-217.

19. Bielinski SJ, Olson JE, Pathak J, Weinshilboum RM, Wang L, Lyke KJ, et al. Preemptive genotyping for personalized medicine: design of the right drug, right dose, right time-using genomic data to individualize treatment protocol. Mayo Clin Proc 2014; 89(1): 25-33.

20. Manolio TA, Chisholm RL, Ozenberger B, Roden DM, Williams MS, Wilson R, et al. Implementing genomic medicine in the clinic: the future is here. Genet Med 2013; 15(4): 258-267.

21.Peissig P, Nikolai A, Glurich I, Brilliant M. Personalized Medicine. In: Hock FJ, editor. Drug Discovery and Evaluation: Pharmacological Assays: Springer International Publishing; 2016. p. 4235-4249.

22.Pulley JM, Denny JC, Peterson JF, Bernard GR, Vnencak-Jones CL, Ramirez AH, et al. Operational implementation of prospective genotyping for personalized medicine: the design of the Vanderbilt PREDICT project. Clin Pharmacol Ther 2012; 92(1): 87-95.

23. Gottesman O, Kuivaniemi H, Tromp G, Faucett WA, Li R, Manolio TA, et al. The Electronic Medical Records and Genomics (eMERGE) Network: past, present, and future. Genet Med 2013; 15(10): 761-771. 
24. Rasmussen-Torvik LJ, Stallings SC, Gordon AS, Almoguera B, Basford MA, Bielinski SJ, et al. Design and anticipated outcomes of the eMERGE-PGx project: a multicenter pilot for preemptive pharmacogenomics in electronic health record systems. Clin Pharmacol Ther 2014; 96(4): 482-489.

25. National Human Genome Research Institute. Clinical Sequencing Exploratory Research (U01). [July 30, 2016]; Available from: http://grants.nih.gov/grants/guide/rfa-files/RFA-HG-10-017.html.

26. Herr T, Bielinski S, Bottinger E, Brautbar A, Brilliant M, Chute C, et al. Practical considerations in genomic decision support: The eMERGE experience. J Pathol Inform 2015; 6(1).

27. Shirts BH, Salama JS, Aronson SJ, Chung WK, Gray SW, Hindorff LA, et al. CSER and eMERGE: current and potential state of the display of genetic information in the electronic health record. J Am Med Inform Assoc 2015; 22(6): 1231-1242.

28. Tarczy-Hornoch P, Amendola L, Aronson SJ, Garraway L, Gray S, Grundmeier RW, et al. A survey of informatics approaches to whole-exome and whole-genome clinical reporting in the electronic health record. Genet Med 2013; 15(10): 824-832.

29. Health Level Seven (HL7) International. Clinical Genomics. 2016 [July 30, 2016]; Available from: http://www.hl7.org/Special/committees/clingenomics/index.cfm.

30. Siu LL, Lawler M, Haussler D, Knoppers BM, Lewin J, Vis DJ, et al. Facilitating a culture of responsible and effective sharing of cancer genome data. Nat Med 2016; 22(5): 464-471.

31. Overby CL, Rasmussen LV, Hartzler A, Connolly JJ, Peterson JF, Hedberg RE, et al. A Template for Authoring and Adapting Genomic Medicine Content in the eMERGE Infobutton Project. AMIA Annu Symp Proc 2014; 2014: 944-953.

32. Heale B, Overby C, Del Fiol G, Rubinstein W, Maglott D, Nelson T, et al. Integrating Genomic Resources with Electronic Health Records using the HL7 Infobutton Standard. Appl Clin Inform [In press]. 2016.

33. Cimino JJ, Jing X, Del Fiol G. Meeting the electronic health record „meaningful use“ criterion for the HL7 infobutton standard using OpenInfobutton and the Librarian Infobutton Tailoring Environment (LITE). AMIA Annu Symp Proc 2012; 2012: 112-120.

34. Alterovitz G, Warner J, Zhang P, Chen Y, Ullman-Cullere M, Kreda D, et al. SMART on FHIR Genomics: facilitating standardized clinico-genomic apps. J Am Med Inform Assoc 2015; 22(6): 1173-1178.

35. Institute of Medicine. Representing Genomic Information in the EHR Ecosystem. Genomics-enabled learning health care systems: Gathering and using genomic information to improve patient care and research: Workshop summary. Washington, DC: The National Academies Press; 2015.

36. Rohrer Vitek CR, Nicholson WT, Schultz C, Caraballo PJ. Evaluation of the use of clinical decision support and online resources for pharmacogenomics education. Pharmacogenomics 2015; 16(14): 1595-1603.

37.Del Fiol G, Huser V, Strasberg HR, Maviglia SM, Curtis C, Cimino JJ. Implementations of the HL7 Context-Aware Knowledge Retrieval („Infobutton“) Standard: challenges, strengths, limitations, and uptake. J Biomed Inform 2012; 45(4): 726-735.

38. CDS Hooks - Home. 2015 [July 30, 2016]; Available from: https://github.com/cds-hooks/cds-hooks/wiki.

39. Overby CL, Heale B, Aronson S, Cherry JM, Dwight S, Milosavljevic A, et al. Providing Access to Genomic Variant Knowledge in a Healthcare Setting: A Vision for the ClinGen Electronic Health Records Workgroup. Clin Pharmacol Ther 2016; 99(2): 157-160.

40. Goel MS, Brown TL, Williams A, Cooper AJ, Hasnain-Wynia R, Baker DW. Patient reported barriers to enrolling in a patient portal. J Am Med Inform Assoc 2011; 18(Suppl. 1): i8-i12.

41.Davis SE, Osborn CY, Kripalani S, Goggins KM, Jackson GP. Health Literacy, Education Levels, and Patient Portal Usage During Hospitalizations. AMIA Annu Symp Proc 2015; 2015: 1871-1880.

42.Irizarry T, DeVito Dabbs A, Curran CR. Patient Portals and Patient Engagement: A State of the Science Review. J Med Internet Res 2015; 17(6): e148.

43.43. Stuckey H, Williams JL, Fan AL, Rahm AK, Green J, Feldman L, et al. Enhancing genomic laboratory reports from the patients' view: A qualitative analysis. Am J Med Genet A 2015; 167a(10): 2238-2243.

44. Williams J, Rahm A, Stuckey H, Green J, Feldman L, Zallen D, et al. Enhancing Genomic Laboratory Reports: A Qualitative Analysis of Provider Review. Am J Med Genet A 2016; 170(5): 1134-1141. 